\title{
Search for Charged Lepton Flavor Violation at the Mu2e Experiment
}

\author{
Markus Röhrken*† \\ California Institute of Technology \\ E-mail: roehrkenecaltech.edu
}

The Mu2e experiment being constructed at Fermilab will search for the coherent neutrino-less conversion of muons to electrons in the field of an atomic nucleus. Mu2e will probe for this charged lepton flavor violating process at a sensitivity of a few parts in $10^{-17}$, an improvement of four orders of magnitude compared to previous experiments, providing a unique probe for physics beyond the Standard Model up to the $10^{4} \mathrm{TeV}$ scale. This proceedings article addresses the underlying physics including the prospective sensitivity to physics beyond the Standard Model and the experimental setup.

Flavor Physics \& CP Violation 2015,

May 25-29, 2015

Nagoya, Japan

*Speaker.

†n behalf of the Mu2e Collaboration. 


\section{Introduction}

Although not forbidden, charged lepton flavor violating (CLFV) processes are extremely suppressed in the Standard Model (SM) due to the tiny neutrino mass. For example, the SM rate for the neutrino-less decay of a free muon into an electron and photon relative to the ordinary muon decay is

$$
\frac{\Gamma(\mu \rightarrow e \gamma)}{\Gamma(\mu \rightarrow e v v)} \propto\left|\sum_{i} \frac{m_{v_{i}}^{2}}{m_{W}^{2}} U_{\mu i}^{*} U_{e i}\right|^{2} \approx 10^{-54},
$$

which is far below any conceivable experimental sensitivity.

However many models of physics beyond the SM predict significantly enhanced CLFV rates. These models include lepto-quarks, heavy neutrinos, GUTs, extra dimensions, little Higgs or diverse variations of supersymmetry. Any experimental detection of a CLFV signal would point to new physical phenomena. A review of extensions of the SM and the experimental prospects for CLFV can be found in Reference [1].
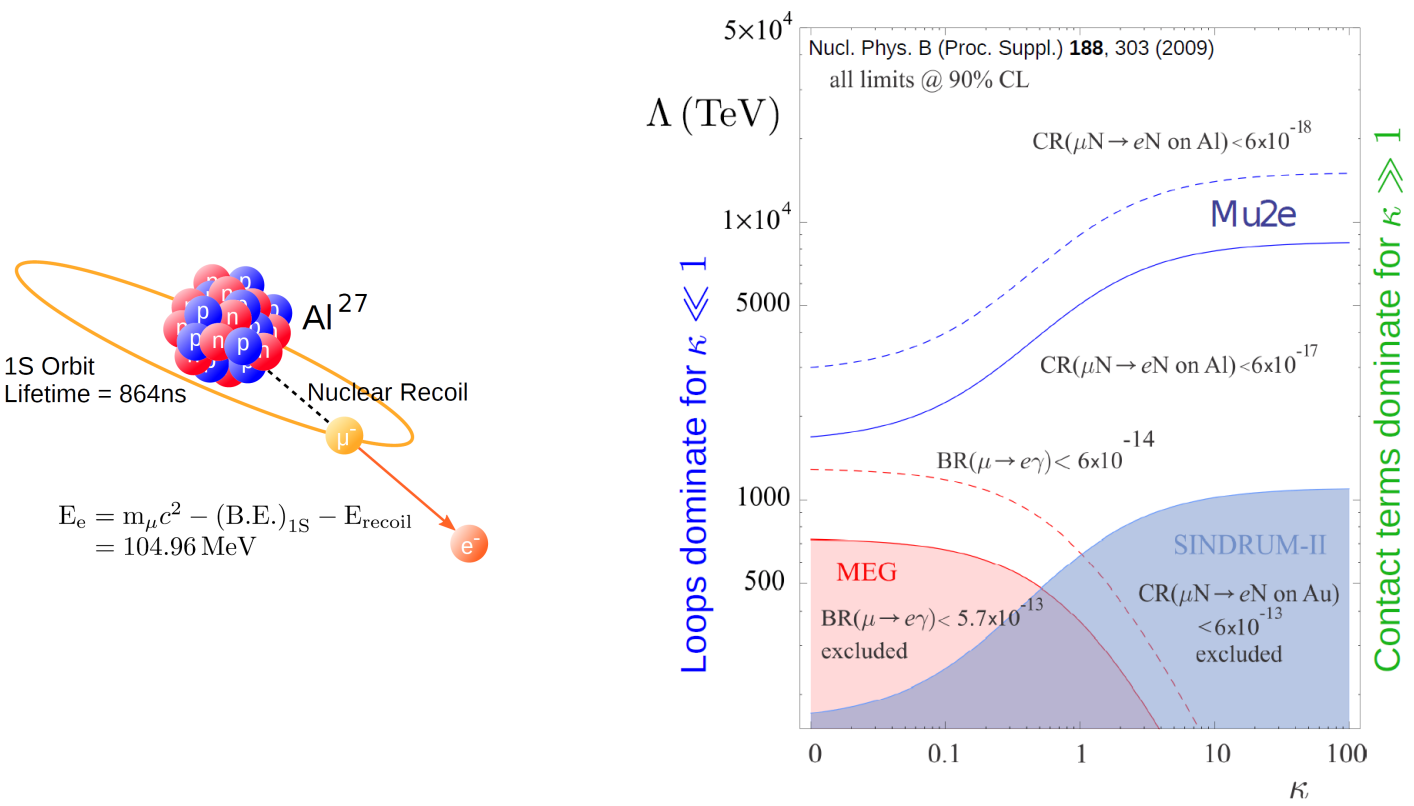

Figure 1: (Left) Neutrino-less conversion of muons to electrons in the field of an Aluminum nucleus. (Right) Sensitivity of CLFV to physics beyond the SM [2].

An important class of CLFV processes is provided by the neutrino-less conversion of muons to electrons in the field of an atomic nucleus. The $\mu N \rightarrow e N$ conversion is a coherent process resulting from an interaction with a nearby nucleus. An illustration of the conversion of a muon bound in the 1s state of an aluminum nucleus is shown in Figure 1 (left). Corrected for the nuclear recoil and the binding energy in the muonic aluminum atom, the experimental signature for the neutrino-less conversion is a single $105 \mathrm{MeV}$ electron.

Various experiments have searched for CLFV. The most stringent upper limits come from $\operatorname{MEG}\left(\mathscr{B}(\mu \rightarrow e \gamma)<5.7 \times 10^{-13}\right)[3]$ and SINDRUM II $\left(C R(\mu N \rightarrow e N\right.$ on Au $\left.)<6 \times 10^{-13}\right)$ [4]. Figure 1 (right) shows the sensitivity of the $\mu \rightarrow e \gamma$ and $\mu N \rightarrow e N$ processes to the effective mass scales of possible beyond the SM physics in a model-independent approach as a function of a relative strength parameter $\kappa$. The $\mu \rightarrow e \gamma$ and $\mu N \rightarrow e N$ processes have complementary sensitivity to physics beyond the SM. For example $\mu \rightarrow e \gamma$ is more sensitive to dipole interactions $(\kappa \ll 1)$, while $\mu N \rightarrow e N$ is more sensitive to contact interactions ( $\kappa \gg 1)$. It is important to experimentally search for both CLFV processes to be able to disentangle the underlying physics. 
Mu2e will search for CLFV in muonic aluminum atoms and aims for a $\mu^{-} N \rightarrow e^{-} N$ singleevent sensitivity of $2.5 \times 10^{-17}$ relative to the ordinary $\mu^{-} N \rightarrow v_{\mu} N^{\prime}$ nuclear muon capture. This is an improvement in sensitivity of four orders of magnitude compared to SINDRUM II and enables to probe for beyond the SM physics up to the $10^{4} \mathrm{TeV}$ scale.

\section{The Experimental Setup}

An illustration of the experimental setup of the Mu2e experiment is shown in Figure 2. The Mu2e experiment comprises three large connected superconducting solenoids: a production solenoid, which captures and focuses particles from the interactions of the incident proton beam on a tungsten target mounted within that solenoid; an s-shaped transport solenoid, which transfers low energy muons and contains collimators for charge and momentum selection; and a large volume detector solenoid, which accommodates the muon stopping target, the tracker and the electromagnetic calorimeter. The solenoid volumes are evacuated. The solenoids provide a graded magnetic field ranging from 4.6 $\mathrm{T}$ upstream in the production solenoid to $1 \mathrm{~T}$ downstream in the detector solenoid.

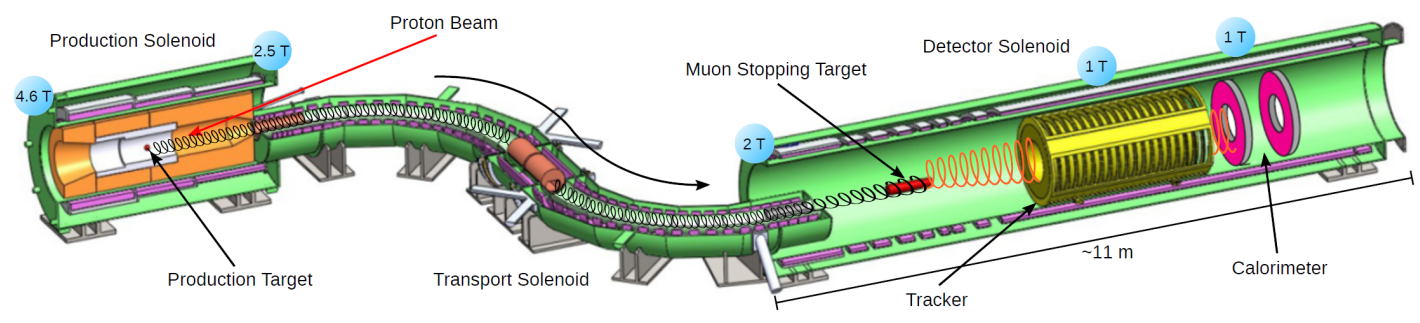

Figure 2: Cut away view of the Mu2e experiment highlighting internal components.

An aluminum stopping target is placed central in the detector solenoid to slow down, stop and then capture muons to form muonic atoms with the target material. A tracker is placed behind the stopping target. It consists of a low mass array of about 20,000 straw tubes of $5 \mathrm{~mm}$ diameter to measure the trajectories of charged particles inside an uniform magnetic field. The tracker is $3 \mathrm{~m}$ long and has an inner hole to be insensitive to charged particles with momenta less than about $55 \mathrm{MeV} / c$, which can originate from the beam or from Michel decays in the stopping target. The tracker is the primary active detector component and measures the momenta from the trajectories of charged particles. An electromagnetic calorimeter is placed downstream of the tracker. It consists of two disks, each composed of about $900 \mathrm{BaF}_{2}$ scintillating crystals each read out by two avalanche photodiodes. The electromagnetic calorimeter contributes to the particle identification and the trigger, and can independently confirm measurements by the tracker. Further important components of the Mu2e experiment are a cosmic ray veto, several proton absorbers, a muon beam stop and monitor systems for the beam and the stopping target. A detailed description of the Mu2e experiment is provided in Reference [5].

\section{Backgrounds and Detection of the Conversion Signal}

Muons stopped in the aluminum target cascade down to the 1s state under X-ray emission. The 1s state has a lifetime of $864 \mathrm{~ns}$, and $61 \%$ of the muons are captured by the nucleus, while $39 \%$ of the muons decay in orbit (DIO) to electrons. Electrons originating from DIOs form the dominant irreducible background of the Mu2e experiment. The energy spectrum of DIO electrons follows essentially the Michel spectrum of the free muon decay with most electrons emitted at energies below $53 \mathrm{MeV}$. Due to recoil effects with the nucleus, the DIO background has a highenergy tail extending the electron spectrum up to the endpoint given by the conversion energy. Close to the endpoint, the DIO spectrum falls rapidly, approximately as $\left(E_{\text {endpoint }}-E_{e}\right)^{5}$ [6]. For the design luminosity of the Mu2e experiment corresponding to $3.6 \times 10^{20}$ protons-on-target and 
$6.7 \times 10^{17}$ stopped muons, 0.20 DIO background events are expected in the signal region. Other small contributions to the background originate from sources such as radiative pion capture, muon and pion decays in flight, or from processes induced by cosmic rays and antiprotons.
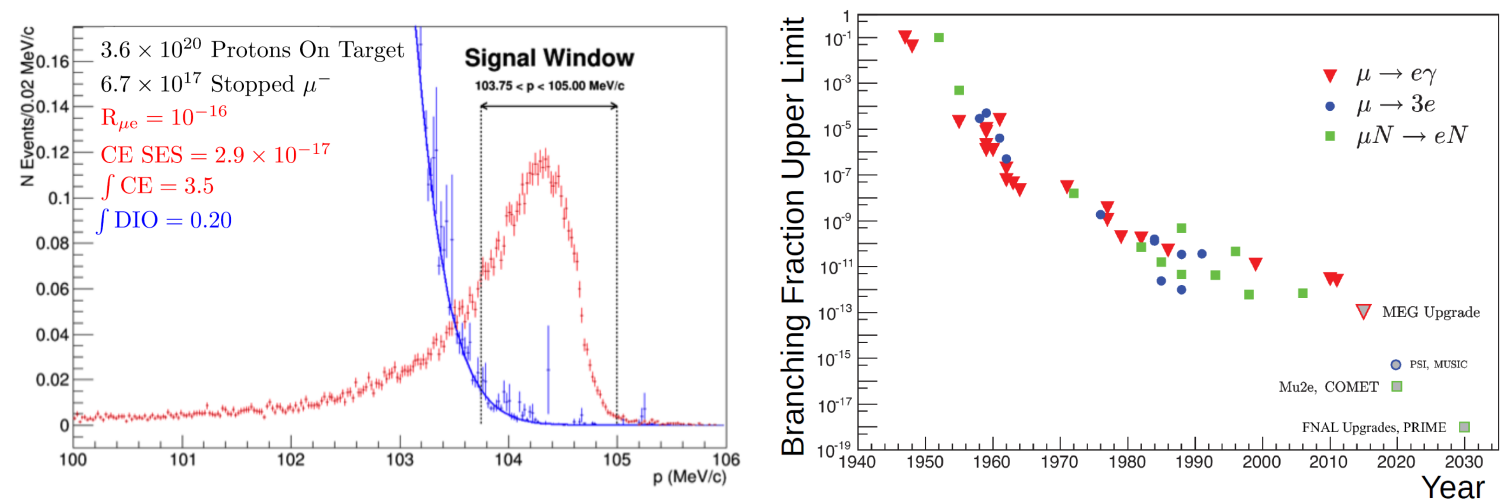

Figure 3: (Left) Reconstructed simulated momentum spectra for electrons from decay-in-orbits (blue) and conversion electrons (red). (Right) Prospects of experimental searches for charged lepton flavor violation [7].

The experimental signature for the $\mu^{-} N \rightarrow e^{-} N$ conversion is a mono-energetic electron populating the signal region at the endpoint energy. The separation of the signal from the background requires an excellent momentum resolution of the tracker better than a few hundred $\mathrm{keV} / c$. Figure 3 (left) shows a reconstructed electron momentum spectrum obtained from simulations including background assuming a conversion rate of $R_{\mu e}=10^{-16}$ and the design luminosity of Mu2e.

\section{Summary}

Mu2e is currently being constructed at Fermilab. The commissioning of the experiment is scheduled for the year 2020. With the COMET experiment being built at J-PARC [8,9] and other possible future facilities, Mu2e will probe for the neutrino-less conversion of muons to electrons at unprecedented precision. The prospects of experimental searches for CLFV are shown in Figure 3 (right).

\section{References}

[1] S. Mihara, J.P. Miller, P. Paradisi, G. Piredda, Annu. Rev. Nucl. Part. Sci. 63, 531 (2013).

[2] A. de GouvÃła, Nucl. Phys. B (Proc. Suppl.) 188, 303 (2009).

[3] J. Adam et al. (MEG Collaboration), Phys. Rev. Lett. 110, 201801 (2013).

[4] W. Bertl et al. (SINDRUM-II Collaboration), Eur. Phys. J. C 47, 337 (2006).

[5] L. Bartoszek et al. (Mu2e Collaboration), "Mu2e Technical Design Report", Fermilab-TM-2594, arXiv:1501.05241 (2015).

[6] A. Czarnecki, X. Garcia i Tormo, W. Marciano, Phys. Rev. D 84, 013006 (2011).

[7] R.H. Bernstein, P.S. Cooper, Phys. Rept. 532, 27 (2013).

[8] Y.G. Cui et al. (COMET Collaboration), “COMET Conceptual Design Report”, KEK-2009-10 (2009).

[9] R. Akhmetshin et al. (COMET Collaboration), "COMET Phase-I Technical Design Report", KEK-2015-1 (2015). 